\title{
Paragonimiasis mimicking chest cancer and abdominal wall metastaisis: A case report
}

\author{
RONGXING ZHOU ${ }^{1 *}$, MINJIA ZHANG ${ }^{2 *}$, NANSHENG CHENG $^{1}$ and YONG ZHOU ${ }^{1}$ \\ Departments of ${ }^{1}$ Biliary Surgery and ${ }^{2}$ Cardiology, West China Medical Center of Sichuan University, \\ Chengdu, Sichuan 610041, P.R. China
}

Received December 26, 2014; Accepted February 1, 2016

DOI: $10.3892 / \mathrm{ol} .2016 .4434$

\begin{abstract}
Typical human paragonimiasis demonstrates an elevated eosinophil count, positive immunoblot, nodular shadows of the lung and pleural thickening with pleural effusion, and these symptoms may be confused with chest cancer. In the present case, a rare case of human paragonimiasis mimicking chest cancer and abdominal wall metastasis is described, the 39-year-old male patient was admitted in our hospital for cough, weight loss $5 \mathrm{~kg}$ and a firm mass in right upper abdominal wall. The laboratory test showed unremarkable hematology and biochemistry results. Chest X-ray, Plain computed tomography of the chest and abdomen showed right pleural effusion, several nodules in right lower lung and a mass in the right upper abdominal wall. The initial diagnosis was lung or chest cancer with abdominal wall metastasis, and the abdominal wall mass was resected for the final diagnosis. The biopsy revealed eosinophilic granuloma with Charcot-Leyden crystal formation infiltrated in the muscular fibers. Subsequent to assessment of the antibodies against parasites, the final diagnosis of paragonimiasis was made.
\end{abstract}

\section{Introduction}

With the improvement of health conditions, the incidence of parasitic diseases has been reducing worldwide in recent decades (1). Pleuropulmonary paragonimiasis is a food-borne zoonosis commonly caused by the trematode Paragonimus westermani. The life cycle of $P$. westermani involves numerous mammals, including humans (2). In humans, the adult worms usually infiltrate the lungs and are found less frequently in other organs, such as the brain and heart (3). The

Correspondence to: Professor Nansheng Cheng, Department of Biliary Surgery, West China Medical Center of Sichuan University, 37 Guo Xue Vally, Chengdu, Sichuan 610041, P.R. China

E-mail: zhourongxing@163.com

*Contributed equally

Key words: paragonimiasis, lung cancer, biopsy, abdominal wall metastasis eggs are expelled from the pulmonary system through the bronchioles and are expectorated or swallowed and passed in the feces, eventually reaching freshwater, such as ponds, streams or rivers, and the next life-cycle is commenced (4). The typical symptoms include pleural effusions and pulmonary nodules, with the most notable clinical features including coughing, blood-tinged sputum and hemoptysis, distressing chest pain and dyspnea (3). However, this parasitic infection has other diverse symptoms and may mimic other conditions, such as tuberculosis infection or neoplasms (5). A range of compounds have been tested for their efficacy against paragonimiasis. Praziquantel and triclabendazole are the two World Health Organization-recommended drugs, and praziquantel is the drug most commonly used in China (6). The majority of the patients have a good prognosis. Approximately 50 species of paragonimiasis have been described worldwide, including 38 species in China, among which the most predominant infections are P. westermani (7). Despite this, it is extremely rare that human paragonimiasis with abdominal wall invasion occurs. In the present study, the case of a patient that was finally diagnosed with human paragonimiasis that mimicked chest cancer with abdominal wall metastasis is reported.

\section{Case report}

In March 2014, a 39-year-old emaciated male patient was admitted to West China Medical Center (Chengdu, Sichuan, China) with a cough and weight loss of $5 \mathrm{~kg}$ in one month. The patient had no symptoms of fever, night sweats or hemoptysis, but did possess a history of iodine allergy, mild alcohol use and heavy smoking. The patient provided written informed consent for the publication of the present study and the study was approved by the Ethics Committee of the West China Hospital, Sichuan University (Chengdu, China).

On examination, the patient demonstrated decreased breathing sounds in the right lower lung, with pleural rub. A firm mass in the right upper abdominal wall was palpable with slight tenderness. Blood tests showed a normal leukocyte count $\left(6.1 \times 10^{9} / 1\right.$; normal range, $\left.3.5-9.5 \times 10^{9} / 1\right)$ and eosinophil granulocyte level of $4.2 \%$ (normal range, $0-5 \%$ ), with normal liver and renal function. Tuberculosis testing was negative. Chest X-ray (Precision Thunis 800+; GE Healthcare, Chalfont, UK) and 32-slice plain computed tomography (Definition AS; Siemens, Munich, Germany) of the chest and abdomen showed 




Figure 1. Computed tomography showing pleural effusion (yellow arrow) and a mass in the abdominal wall (red arrow).

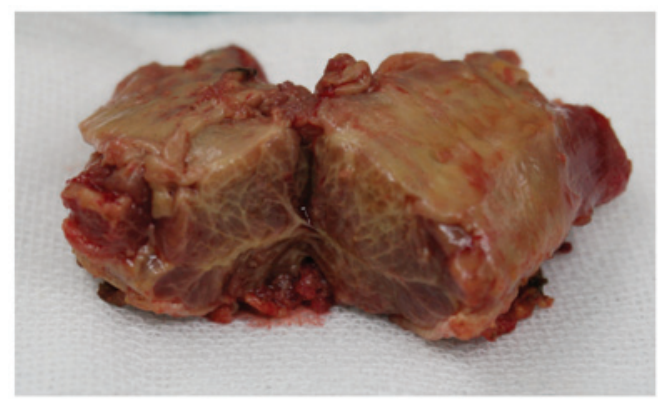

Figure 2. Following resection, the mass was identified as edematous and having fragile rectus abdominis, with a greyish section.

right pleural effusion, several nodules in right lower lung and a mass located in right upper abdominal wall (Fig. 1). Based on these findings, the patient was initially diagnosed with lung or pleural carcinoma with abdominal wall metastasis. To clarify the diagnosis, abdominal wall mass excision was performed under local anesthesia. Intraoperatively, the mass was identified as edematous and fragile rectus abdominis, with a greyish section (Fig. 2). Biopsy specimens were sent to the Department of Pathology (West China Hospital) for pathological analysis, which revealed that the muscle fibers were infiltrated by eosinophilic granuloma with the Charcot-Leyden crystal formation (Fig. 3). The patient was tested for antibodies against parasites, and the result revealed the presence of antibodies against $P$. westermani immunoglobulin $(\mathrm{Ig}) \mathrm{G}$. The patient was diagnosed with $P$. westermani infection. Following $1.5 \mathrm{~g}$ t.i.d. of oral praziquantel treatment for 2 days, the pleural effusion disappeared subsequent to 1 month, and the patient was without recurrence subsequent to 8 months of follow-up.

\section{Discussion}

Only a small number of cases of human paragonimiasis have been described in the English and Japanese medical literature since 1984 (8-10). To the best of our knowledge, only two cases in the Japanese literature have reported a mass in the abdominal wall, although paragonimiasis may invade the abdominal wall muscle for a transient time in its life-cycle in humans $(2,3)$.
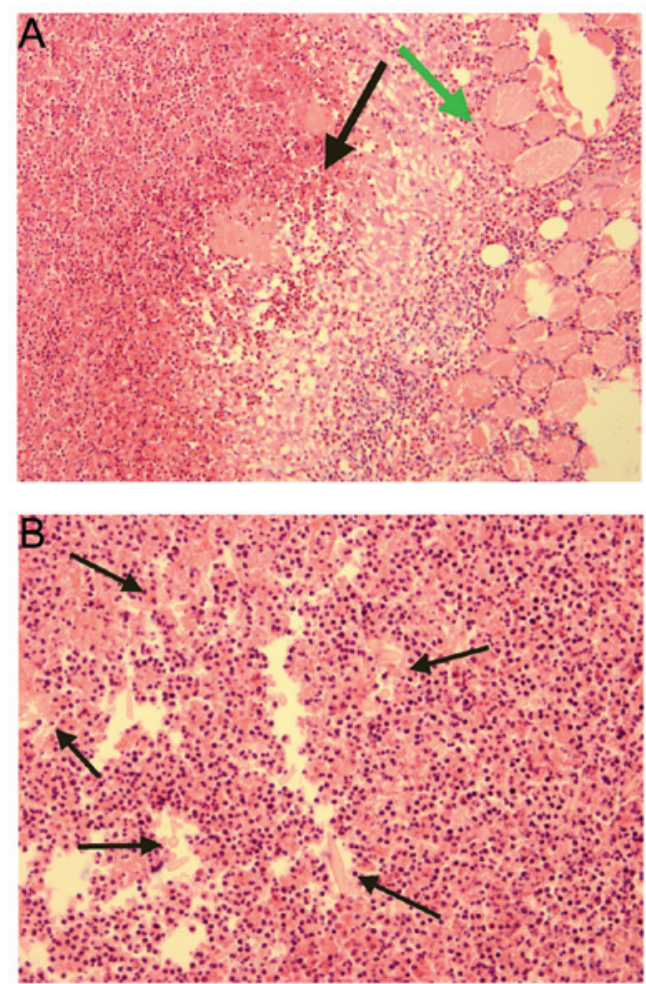

Figure 3. Biopsy showing (A) muscle fibres (green arrow) infiltrated with eosinophilic granuloma (black arrow) and (B) the formation of Charcot-Leyden crystals.

When humans ingest pickled or uncooked food containing an infected crustacean host, such as crabs, crayfish or snails, excystation of the metacercariae occurs in the small intestine and the metacercariae penetrate the intestinal wall and enters the peritoneal cavity, migrate to the diaphragm, pleura and finally reach the lung (3). The central nervous system is the most common site of extrathoracic paragonimiasis (3).

Typical human paragonimiasis demonstrates an elevated eosinophil count, positive immunoblot, nodular shadows of the lung and pleural thickening with pleural effusion, and these symptoms may be confused with tuberculosis or chest cancer (4). In the present study, the patient had lung nodules and small amount of pleural effusion with a normal eosinophil granulocyte level. In addition, due to a mass in the abdominal wall of the patient and history of weight loss, the infection was not distinguished from chest cancer and abdominal wall metastasis. If the patient had possessed cancer with advanced metastasis, the opportunity for surgery would have been lost and the patient would receive a poor prognosis (11). To clarify the diagnosis and prepare for the following treatment, a biopsy of the abdominal wall mass with surgical resection was performed. Therefore, surgical intervention is possible in certain cases for the diagnosis and treatment of paragonimiasis. The biopsy indicated that the patient suffered from parasite infection, and the final diagnosis and blood test for P.westermani $\mathrm{IgG}$ antibodies were performed. Corresponding history, antibody tests and biopsy contribute the convincing evidence for the correct diagnosis (12).

Praziquantel can damage the cortex of parasites and stimulate muscle tonic contraction, causing spastic paralysis and death, and is recommended as an orally administered agent 
at a total dose of $75-150 \mathrm{mg} / \mathrm{kg}$ (13). The cure rate is $80-90 \%$, with infrequent side effects (14). It should be considered that insufficient dosage may lead to a worse outcome. Other drugs, such as mebendazole and bithionol, have been investigated as experimental chemotherapies for paragonimiasis. Praziquantel is orally administered at a total dose of $150 \mathrm{mg}$ per $\mathrm{kg}$ of body weight, and is divided into three doses per day for 2 days. The cure rates reported are $80-90 \%$. Mebendazole plus emetine hydrochloride shows a cure rate of $70 \%$, while bithionol achieves only a 50-60\% cure rate, and side effects such as urticaria, rash, abdominal pain, nausea, vomiting, diarrhea and dizziness are common $(15,16)$. In the present case, the patient achieved a good recovery subsequent to a course of oral $150 \mathrm{mg} / \mathrm{kg}$ praziquantel treatment, which was divided into three doses per day for 2 days, and did not experience symptoms of recurrence within 8 months.

The present study emphasizes the requirement for the consideration of paragonimiasis when a patient presents with lung nodules and pleural effusion, even without an elevated eosinophil count. Paragonimiasis may mimic chest cancer and abdominal wall metastasis. Clinical history, blood antibody and imaging studies are important to make a correct diagnosis. Biopsy is particularly important in selected cases to exclude other causes, such as neoplasm and tuberculosis.

\section{References}

1. Ahn MH: Changing patterns of human parasitic infection in Korea. Hanyang Med Rev 30: 149-155, 2010.

2. Vélez I, Velásquez LE and Vélez ID: Morphological description and life cycle of Paragonimus sp. (Trematoda: Troglotrematidae): Causal agent of human paragonimiasis in Colombia. J Parasitol 89: 749-755, 2003.

3. Liu Q, Wei F, Liu W, Yang S and Zhang X: Paragonimiasis: An important food-borne zoonosis in China. Trends Parasitol 24: 318-323, 2008.
4. Kagawa FT: Pulmonary paragonimiasis. Semin Respir Infect 12: 149-158, 1997.

5. Jeon K, Koh WJ, Kim H, Kwon OJ, Kim TS, Lee KS and Han J: Clinical features of recently diagnosed pulmonary paragonimiasis in Korea. Chest 128: 1423-1430, 2005.

6. Chen MG: New developments in the clinical study of praziquantel. Chin J Parasitol Parasit Dis 2: 193-195, 1984.

7. Zhang ZH: Paragonimiasis. In: Diseases of Natural Focus. Tang JQ (ed). Chinese Science Press, Beijing, pp1085-1098, 2005.

8. Akaba T, Takeyama K, Toriyama M, Kubo A, Mizobuchi R, Yamada T, Tagaya E, Kondo M, Sakai S and Tamaoki J: Pulmonary paragonimiasis: The detection of a worm migration track as a diagnostic clue for uncertain eosinophilic pleural effusion. Intern Med 55: 503-506, 2016.

9. Yang X, Xu M, Wu Y and Xiang B: Pancreatic paragonimiasis mimics pancreatic cystic-solid tumor - A case report. Pancreatology 15: 576-578, 2015.

10. Hoshina T, Tamura K, Kawano S, Kato T, Sato F, Horino T, Nakazawa Y, Yosikawa K, Yoshida M, Kumagai M and Hori S: Two cases of Paragonimiasis westermani in a Chinese family diagnosed with the Ouchterlony double diffusion method. Kansenshogaku Zasshi 88: 866-870, 2014 (In Japanese).

11. Bagcchi S: Use of chemotherapy in patients with metastatic lung cancer. Lancet Oncol 16: e263, 2015.

12. Castilla EA, Jessen R, Sheck DN and Procop GW: Cavitary mass lesion and recurrent pneumothoraces due to Paragonimus kellicotti infection: North American paragonimiasis. Am J Surg Pathol 27: 1157-1160, 2003.

13. Johnson RJ, Jong EC, Dunning SB, Carberry WL and Minshew BH: Paragonimiasis: Diagnosis and the use of praziquantel in treatment. Rev Infect Dis 7: 200-206, 1985.

14. Drugs for Parasitic Infections. 2nd edition. The Medical Letter, Inc., New Rochelle, NY, pp1-20, 2010.

15. Benjapong W, Naeypatimanond S, Benjapong K, Thumaruksa C, Ruttarasarn S and Jaroonvesama N: Studies on paragonimiasis: Treatment with mebendazole, emetine with mebendazole and praziquantel. Southeast Asian J Trop Med Public Health 15: 354-359, 1984.

16. Chen MG, Chang ZS, Shao XY, Liu MD, Blair D, Chen SH, Zhang YN, Hong JL, Shen BG and Feng Z: Paragonimiasis in Yongjia County, Zhejiang Province, China: Clinical, parasitological and karyotypic studies on Paragonimus westermani. Southeast Asian J Trop Med Public Health 32: 760-769, 2001. 